\title{
A Discussion of Sources and Description of the Earth's Magnetic Field and Its Secular Variations
}

\author{
Leroy R. Alldredge and Charles O. Stearns \\ U.S. Geological Survey, Boulder, Colorado, U.S.A. \\ (Received May 20, 1974; Revised July 8, 1974)
}

\begin{abstract}
The problem of collecting data for making geomagnetic charts including secular change is reviewed. Satellite data gives excellent coverage, but the satellite total field intensity data is not sufficient to properly define the field components. In constructing charts, the specific time and space filtering that is used should be specified so the user can make needed corrections in the use of the charts. An attempt should be made to include time variations having periods of one year or greater. In the space domain spherical harmonic coefficients up to degree 12 or 13 should probably be included. Adequate charts in the future will likely require the use of some external coefficients.
\end{abstract}

\section{General Description of the Problem}

A need to know the magnetic field vector all over the surface of the earth and in the region surrounding the earth is related to survey, resource, navigation, and space studies. Since instantaneous worldwide surveys are not possible, knowledge of the field now or in the future implies the availability of adequate charts or mathematical models which include accurate information about the secular variation of the earth's magnetic field.

Years ago the data accumulated so slowly that a good knowledge of the secular-change over a considerable period of time was required to bring the observed data up to the epoch chosen for the chart. All official world charts published by the United Kingdom, the United States, and the U.S.S.R. show constant secular-change rates which are to be used throughout the life of the chart.

By means of magnetometers in satellites, enough data for a chart analysis may now be collected quickly enough so that correction for secular-change is of secondary importance. However, so far only total field intensity has been obtained from satellites for charting purposes. Fabiano and Peddie (1971) found that the secular change of vertical intensity at some equatorial observatories was poorly fitted by the satellite $F$ (total field intensity) model POGO (8/69). Hurwitz (1972) showed that a model constructed from noisy $F$ data fails to give the correct vertical intensity $(Z)$ in the vicinity of the dip equator. For this reason, is appears that component data are still very desirable. We hope that such data can be obtained from satellites in the not-too-distant future. Until that time there is still a need for good ground data. 
Although the quick collection of data makes corrections for secular-change unimportant, surveys extended in time will be necessary to obtain the secularchange itself, so it can be included in the charts and in models.

The component ground data or aircraft data in some cases still suffer from very irregular spacing that can likely give rise to errors in unsurveyed areas.

Assuming that solutions are attainable for the problems related to the spacing of observations, the speed with which surveys can be made, and the need to have component data, the effects of local crustal anomalies and of external sources will still complicate the analysis and presentation of the results.

Ideally, one would like to have a detailed description of the earth's field which would give a true picture in space and time of the actual field. Such a description, if done in spherical harmonic form, would require four million coefficients to adequately include local anomalies only $20 \mathrm{~km}$ wide. Each of these coefficients should be a function of time, admitting frequencies approaching $1 \mathrm{~Hz}$ if most micropulsations were to be accounted for. Such a picture will likely never be developed and indeed is not needed for any practical purpose.

In the recent past, it has been tacitly assumed that the chart representations of the earth's magnetic field included only sources in the core of the earth. This idea was often conveyed by use of the term "The Earth's Main Magnetic Field". In practice there was really no way to be sure that only contributions from the core were included. The actual observed data were usually smoothed in space and time in different ways by different analysts. The residual, left for display on charts or to be presented by a set of spherical harmonic coefficients, gives a practical and useful result, but it cannot be said that it resulted only from selected specified sources.

Fortunately the problem has some natural divisions in space and time which are useful. In the space domain it appears quite certain that the so-called local anomalies (less than a few hundreds of kilometers in extent) are caused by crustal sources (AlLDREDGE et al., 1963; Bullard, 1967). If we agree that in our representation of the field we will eliminate these local anomalies, then we can greatly reduce the order and degree of the model. The place where one should stop in the space and time filtering is not yet entirely obvious. REGAN (1973) and Kolesova and Kropachev (1973) are searching for harmonic terms above the usual cutoff point which is between order 8 and 12 . They feel these may be caused by sources in the deep crust, and they may end up with expansions up to order and degree 12 to 20 or even 25. Perhaps such expansions will be practicable as the data improve. The higher order terms will of course have small amplitudes, and as soon as we are sure they are real they should be retained.

In the time domain it is even less clear just what we should try to retain. If there are any sizeable variations due to external sources having periods of several months or longer, we may need to account for them somehow in our 
description. If this is done, the end product will no longer be a "description of the earth's main magnetic field"- - meaning from sources internal to the earthinstead it will be a "description of the earth's magnetic field containing periods greater than a stated period," say a month or a year including external sources.

The amplitude of short period pulsations is very small; these pulsations average out to zero in a matter of seconds. Regular daily variations will also tend to average to zero over periods of time of interest to charting, and can probably be neglected along with the pulsations. Individual magnetic storms cannot be predicted, therefore they cannot be included in our description and, in fact, they should not be included because most of the energy is contained in relatively short periods. On the other hand there is no assurance that external sources influenced by the 11- and 22-year sunspot cycles, etc., will not overlap in frequency with variations coming from the core. Hence, as suggested above, our final description may not be restricted to sources in the core.

Limitations as to what part of the time spectrum should be included in a model description of the earth's magnetic field are quite arbitrary and are made only for expediency. It is proposed here that we simply admit the impracticability of including daily and storm time variations, but that we attempt to include periods of 1 year and greater if they are of sufficient amplitude and if we can find out how to predict them in any useful way. If this suggestion were adopted, it would mean that those making field measurements, who intended to use a model description of the earth's field in the reduction of their data, should monitor the daily and storm time variations in the field and should correct their data for these effects before making comparisons with the model description of the field. On the other hand, the experimenters could assume that the model description would account for all longer-period variations. Such a convention would at least delineate responsibilities and goals for those working in the field who are trying to use the charts or models. The description should explicitly state what space and time filtering had been used.

\section{The Space Filter}

Prior to 1965 world magnetic charts were compiled using manual techniques from all available data after secular-change adjustments had been made to reduce the data to a common epoch.

Until about 1945 only point measurements of the field were available. These data came from observatories, repeat stations, and measurements made during special land surveys and ocean cruises. For these early measurements, observation points were typically tens of kilometers apart, even along track lines, and hundreds to thousands of kilometers between observatories. Because of the sparcity of data, it was impossible to include details of crustal anomalies on the charts or for that matter to even appreciate the true nature of these 
narrow anomalies. Although most crustal anomalies were not explicitly shown in the charts, they did have a bearing on the final product.

The draftsman drawing the charts found it difficult to maintain any kind of a constant space filter because of the way the data were presented to him. Examples of this uneven filtering are very evident in the charts by Vestine et al. (1947b). In some areas anomalies as short as $150 \mathrm{~km}$, corresponding to a spherical harmonic of order greater than 200, are retained, whereas in most of the chart only very long wavelengths are evident. This method of constructing charts no doubt left the wrong impression for many areas that were shown as smooth because of lack of data.

Many spherical harmonic analyses (SHA) of the field were made before 1965 (MCDonAld and Gunst, 1967) but most of them were made for research purposes from data taken from hand-drawn charts. Starting in about 1965 the role of SHA was reversed. Since that time the SHA has been made from raw or smoothed observational data and the resulting spherical harmonic coefficients (SHC) have been used to draft the charts. This means that none of the finished charts now show anomalies of finer scale than that corresponding to the highest degree included in the SHA. For the International Geomagnetic Reference Field (IGRF) degree eight was used, which would admit a minimum anomaly wavelength of approximately $5000 \mathrm{~km}$.

The earliest SHA of the earth's field was made by GAuss (1839) for epoch 1835 only to degree four. For many years the SHA's were typically carried out to degree six. The rather low degree used in these early analyses was in part dictated by the sparsity of data, but the limitations of available calculators would have made higher-degree analyses extremely difficult.

As better computers became available and more data were obtained, the question of how high a degree should be included came into focus.

FANSElau et al. (1964) made an analysis of the U.S. world charts of epoch 1945 to degree fifteen. They concluded that if one assumed average errors in the input data as large as $\pm 100 \mathrm{nT}(\mathrm{nT}=$ nanotesla $=1$ gamma) the coefficients above degree six or eight were not useful.

Hurwitz et al. (1966) in constructing charts for epoch 1965, used a SHA to degree twelve. The authors claimed that the higher degree terms are useful in describing the earth's field.

REGAN (1973) has used a model to degree thirteen which he subtracted from observed satellite data to obtain residuals in the total field intensity, with the hope of revealing anomalies in the crust or upper mantle having sources with wavelengths from $5^{\circ}$ to $30^{\circ}$. Regan's analysis has revealed several anomalous areas with amplitudes up to $12 \mathrm{nT}$.

Kolesova and Kropachev (1972) described an integral method which permits the economical computation of SHC to a much higher degree. Kolesova and Kropachev (1973) indicated that by use of this method and with land, sea, 
and airborne vertical component data averaged over $1^{\circ}$ squares, the SHC up to $n=23$ have been computed, but the authors indicated that the coefficients do not differ significantly from zero beyond $n=11$.

AlldREDGe et al. (1963) concluded from an examination of the harmonic spectrum of an "around-the-world" magnetic profile that harmonics greater than degree ten are probably not needed to describe the long wavelength part of the field.

BULLARD (1967) after smoothing the round-the-world spectrum of ALLDREDGE et al. (1963) by convolving it with $1 / 4(1-\cos 2 \pi \eta / 20)$, where $\eta=1$, $2, \ldots 19$, concluded that the spectrum showed a rapid decrease to the 25 th term followed by a much slower downward trend. Kolesova and KROPACHeV (1973) have taken this to mean that the long period part of the field should be carried up to degree 25, which accounts for their analysis up to $\eta=23$.

From the above discussion it is quite clear that there is need for more investigation on where the space filter should have its cutoff. Although arguments have been set forth that a degree as high as 25 should be used and analyses have been carried out to $\eta=23$, the highest degree analysis which seems to have yielded useful results is that reported by REGAN (1973).

\section{Time Variation of External Sources}

One of the severest problems in describing the earth's field is the inadequacy of the available information on secular variation. In 1968 when the first IGRF was agreed upon, there were great differences in the proposed secular change coefficients. For some coefficients, the variations among the proposed models were larger than the agreed-upon coefficients themselves (ZMUDA, 1971).

Most of the significant long-period changes (secular-change) originate in the core of the earth. On the other hand, external sources may be confusing the picture. External sources contribute only a small percentage of the total observed field, but the observed secular change in nanotesla per year is comparable numerically to the field values in nanotesla expected from external sources. Since the time constants for external sources are much shorter than those for core sources, the external sources may contribute importantly to the observed overall rate of change in the field. If these frequencies fall within the time filter we choose, they should be included in the description.

Vestine et al. (1947 a, b) apparently fully understood the problem they faced in trying to determine the main field and its secular change. In the two volumes referenced above, over 200 pages (large format) were devoted to eliminating the following problems from the data before the charts were drawn: geomagnetic changes related to the sunspot cycle, annual variations, post-perturbation variations, and solar quiet day variations, Sq. The authors wanted very much to have the residual data represent only internal sources. 
We are unaware of recent efforts, as extensive as that described in the paragraph above, to correct raw data to eliminate external sources. Data from repeat stations usually indicate a correction for $\mathrm{Sq}$, but most surveys admit that no correction for time variations have been made at all. In making modern SHA, almost none of the survey data or observatory data has been corrected for sunspot cycle, storm variations, annual variations, or post-perturbation variations before the data are fed to the computer. In certain studies authors do make special note of some of these factors. For example, DAvis and CAIN (1973) have made a study of the solar disturbance field by removing it from POGO satellite data. Quite often authors will screen data admitting dates only during the periods of low $\mathrm{Kp}$ indices or during the international quiet days.

The uses to which the charts are put seem to demand that the charts contain all the time variations to be encountered except those for which corrections are readily available.

It is usually assumed that it would be best to exclude the Sq and immediate storm time effects from the data used for charts because of their short periods and variability. In using the chart, for example, to remove a regional field from the observed data, an individual should correct for these variations in his raw data before comparing his data with the chart. On the other hand, we suggest that the charts should include in the "secular change" information variations of longer periods such as the annual variations and sunspot cycle variations, along with the changes caused by core sources.

To include these variations in the charts, they must be studied in retrospect to gain a better understanding of them. This should aid in correcting data to a common epoch and hopefully would help predict them a few years in advance. If this is successful they can be explicitly included in the secular-change description to improve the use of the charts in years succeeding the epoch of the chart itself.

\section{The Expected Effects of External Sources}

The spherical harmonic expression for the geomagnetic potential from external sources is of the form

$$
V_{e}=a \sum_{n=1}^{\infty} \sum_{m=0}^{n}\left(\frac{r}{a}\right)^{n} P_{n}^{m}(\cos \theta)\left\{\left(g_{n}^{m}\right)_{e} \cos (m \lambda)+\left(h_{n}^{m}\right)_{e} \sin (m \lambda)\right\}
$$

where $r, \theta$ and $\lambda$ are the usual spherical coordinates, $a$ is the radius of the earth, $P_{n}^{m}(\cos \theta)$ are the quasi-normalized associated Legendre polynomials, and the $\left(g_{n}^{m}\right)_{e}$ and $\left(h_{n}^{m}\right)_{e}$ are the external spherical harmonic coefficients. Noting that the North, East, and Vertical field intensities are $X_{e}=\partial V_{e} / r \partial \theta, Y_{e}=-\partial V_{e} / r \sin \theta \partial \lambda$, and $Z_{e}=\partial V_{e} / \partial r$, respectively, we get: 


$$
\begin{aligned}
& X_{e}=\sum_{n=1}^{\infty} \sum_{m=0}^{n}\left(\frac{r}{a}\right)^{n-1} \frac{\partial P_{n}^{m}}{\partial \theta}(\cos \theta)\left\{\left(g_{n}^{m}\right)_{e} \cos (m \lambda)+\left(h_{n}^{m}\right)_{e} \sin (m \lambda)\right\} \\
& Y_{e}=\sum_{n=1}^{\infty} \sum_{m=0}^{n}\left(\frac{r}{a}\right)^{n-1} \frac{m P_{n}^{m}(\cos \theta)}{\sin \theta}\left\{\left(g_{n}^{m}\right)_{e} \sin (m \lambda)-\left(h_{n}^{m}\right)_{e} \cos (m \lambda)\right\} \\
& Z_{e}=\sum_{n=1}^{\infty} \sum_{m=0}^{n} n\left(\frac{r}{a}\right)^{n-1} P_{n}^{m}(\cos \theta)\left\{\left(g_{n}^{m}\right)_{e} \cos (m \lambda)+\left(h_{n}^{m}\right)_{e} \sin (m \lambda)\right\}
\end{aligned}
$$

For the first-order terms for which $n=1$, using the fact that $P_{1}^{0}(\cos \theta)=\cos \theta$ and $P_{1}^{1}(\cos \theta)=\sin \theta$ we get:

$$
\begin{aligned}
& X_{e}=-\sin \theta\left(g_{1}^{0}\right)_{e}+\cos \theta\left\{\left(g_{1}^{1}\right)_{e} \cos \lambda+\left(h_{1}^{1}\right)_{e} \sin \lambda\right\} \\
& Y_{e}=\left\{\left(g_{1}^{1}\right)_{e} \sin \lambda-\left(h_{1}^{1}\right)_{e} \cos \lambda\right\} \\
& Z_{e}=\cos \theta\left(g_{1}^{0}\right)_{e}+\sin \theta\left\{\left(g_{1}^{1}\right)_{e} \cos \lambda+\left(h_{1}^{1}\right)_{e} \sin \lambda\right\}
\end{aligned}
$$

which define a uniform field inside the source region with a magnitude equal to

$$
\left[\left(X_{e}\right)^{2}+\left(Y_{e}\right)^{2}+\left(Z_{e}\right)^{2}\right]^{1 / 2}=\left[\left(g_{1}^{0}\right)_{e}^{2}+\left(g_{1}^{1}\right)_{e}^{2}+\left(h_{1}^{1}\right)_{e}^{2}\right]^{1 / 2} .
$$

From (5), (6) and (7) it is clear that the field consists of three uniform perpendicular field components having magnitudes $g_{1}^{0}, g_{1}^{1}$, and $h_{1}^{1}$, respectively, along the three cardinal directions of the spherical coordinate system. An external ring current far from the earth would approximate such a first-degree field.

This is a very different type of field than that given by the first-order terms for internal sources which is that of a centered dipole. This immediately raises the question of what will happen if first-degree external sources are present and an attempt is made to fit the data using the least squares method and a functional form which includes only internal sources.

This problem can perhaps be best illustrated by working through a very simple example showing how internal and external spherical harmonic coefficients can be separated in some cases and how confusion may exist in other cases.

First, assume we have observed only a field due to a very distant equatorial ring current. This will give a uniform field about the earth in the direction of the earth's rotational axis. This symmetry simplifies the description without limiting the applicability. The observed field from such a source will be

$$
\begin{aligned}
& X=K \sin \theta \\
& Z=-K \cos \theta .
\end{aligned}
$$

If we try to fit such "observed" data to a magnetic potential function which admits only first-degree terms for internal and external sources (symmetry again assumed so that $g_{1}^{1}=h_{1}^{1}=0$ ) that is,

$$
V=a\left(\frac{a}{r}\right)^{2}\left(g_{1}^{0}\right)_{i} \cos \theta+a\left(\frac{r}{a}\right)\left(g_{1}^{0}\right)_{e} \cos \theta
$$

which gives at $r=a$ 


$$
X_{\text {computed }}=\frac{\partial V}{r \partial \theta}=-\left(g_{1}^{0}\right)_{i} \sin \theta-\left(g_{1}^{0}\right)_{e} \sin \theta=K \sin \theta \quad \text { (observed value) }
$$

and

$$
Z_{\text {computed }}=\frac{\partial V}{\partial r}=-2\left(g_{1}^{0}\right)_{i} \cos \theta+\left(g_{1}^{0}\right)_{e} \cos \theta=-K \cos \theta \quad \text { (observed value) } .
$$

Simultaneous solution of these two expressions gives

$$
\left(g_{1}^{0}\right)_{e}=-K \quad \text { and } \quad\left(g_{1}^{0}\right)_{i}=0
$$

thus correctly showing that all the field comes from external sources.

Similarly, if we assume we have observed only a field from an internal centered dipole along the principal axis, the observed field will be of the form

$$
\begin{aligned}
& X=K \sin \theta \\
& Z=2 K \cos \theta
\end{aligned}
$$

and if we try to fit the data to the calculated values of Eq. (11) we find this time that

$$
\left(g_{1}^{0}\right)_{e}=0 \quad \text { and } \quad\left(g_{1}^{0}\right)_{i}=-K
$$

which correctly shows that all the field comes from internal sources.

If, on the other hand, we should observe a field from an external source such as given by Eq. (9) and try to fit the data to an expression for only firstdegree internal sources we would find on the surface of the earth that

$$
\left.\begin{array}{l}
X_{\text {computed }}=-\left(g_{1}^{0}\right)_{i} \sin \theta=K \sin \theta \quad \text { (observed value) } \\
Z_{\text {computed }}=-2\left(g_{1}^{0}\right)_{i} \cos \theta=-K \cos \theta \quad \text { (observed value) } .
\end{array}\right\}
$$

One expression yields $\left(g_{1}^{0}\right)_{i}=-K$ and the other gives $\left(g_{1}^{0}\right)_{i}=K / 2$.

These are inconsistent because we started with the wrong functional form to fit the observed data. If a least squares solution is made of Eq. (13) we get

$$
\left(g_{1}^{0}\right)_{i}=\frac{K}{5} .
$$

This value of $\left(g_{1}^{0}\right)_{i}$, used to compute $X$ and $Z$ by the left-hand equalities in Eq. (13), will give a very poor fit as illustrated in Fig. 1 . Note that $X$ has the wrong sign throughout the entire range of $\theta$. The least squares will always force an answer if the problem is at all well behaved, but the result may not be very meaningful if an improper functional form is used in the process. In practice, a more proper functional form than that given in the example above is usually chosen, so that such a poor fit will not generally be encountered. Nevertheless, if the raw data contain external sources (as we know they do) and we try to fit them to functional forms typical only of internal sources (which is usually done) improper coefficients will be derived. 


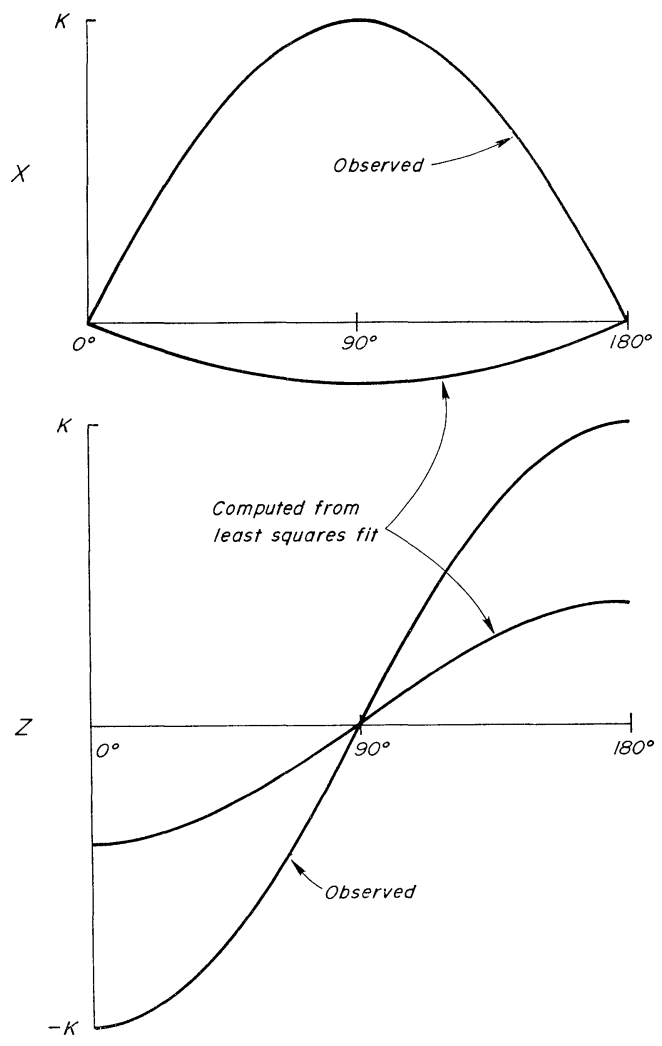

Fig. 1. Comparison of first-degree "observed" field from external sources with least-squares fit to that field using only first-degree internal source model. $X$ is northward horizontal component and $Z$ is the vertical component.

Some additional tests were made with more realistic data to see what would happen if the proper functional forms were not used. A field was first synthesized from the IGRF coefficients at $10^{\circ}$ intervals of latitude and longitude. A uniform field of $100 \mathrm{nT}$ in the direction of the IGRF dipole axis was added to the IGRF field values. This added external source field corresponds to a distant ring current having external spherical harmonic coefficients of

$$
\left(g_{1}^{0}\right)_{e}=98 \mathrm{nT}, \quad\left(g_{1}^{1}\right)_{e}=7 \mathrm{nT} \quad \text { and } \quad\left(h_{1}^{1}\right)_{e}=-19 \mathrm{nT} .
$$

Spherical harmonic analyses were carried out on this composite field under a variety of conditions with the results shown in Table 1 . When the first three external coefficients were admitted in the analysis, all the coefficients including the three external ones were exactly recovered within the limits of precision of the computer, regardless of the weights or form of analysis used $(X, Y$ and $Z$ or $F$ only). This was expected. When the external coefficients were omitted from the solution there were large differences in the results, depending on the 
Table 1. Effect of ring current (added to the IGRF field) and the method of analysis when only the 80 internal IGRF coefficients are used in the analysis. ${ }^{11}$

Changes in spherical harmonic coefficients from the original IGRF coeff $(n T)^{21}$

\begin{tabular}{|c|c|c|c|c|c|c|c|c|c|}
\hline \multirow{2}{*}{$\begin{array}{l}\text { Internal } \\
\text { coeffi- } \\
\text { cients }\end{array}$} & \multicolumn{2}{|c|}{ Weight $=1$} & \multicolumn{2}{|c|}{ Weight $=\sin \theta$} & \multirow{2}{*}{$\begin{array}{l}\text { Internal } \\
\text { coeffi- } \\
\text { cients }\end{array}$} & \multicolumn{2}{|c|}{ Weight $=1$} & \multicolumn{2}{|c|}{ Weight $=\sin \theta$} \\
\hline & $\begin{array}{c}X Y Z \\
\text { Analy- } \\
\text { sis }\end{array}$ & $\underset{\substack{\text { sis } \\
\text { Analy- }}}{F}$ & $\begin{array}{c}X Y Z \\
\text { Analy- } \\
\text { sis }\end{array}$ & $\underset{\substack{\text { Analy } \\
\text { sis }}}{F}$ & & $\begin{array}{c}X Y Z \\
\text { Analy- } \\
\text { sis }\end{array}$ & $\underset{\substack{\text { Analy- } \\
\text { sis }}}{F}$ & $\begin{array}{c}X Y Z \\
\text { Analy- } \\
\text { sis }\end{array}$ & $\begin{array}{c}F \\
\text { Analy- } \\
\text { sis }\end{array}$ \\
\hline$g_{1}^{0}$ & -16 & -4 & (2) & -3 & $h_{5}^{1}$ & & -8 & (2) & -8 \\
\hline$g_{1}^{1}$ & 1 & -5 & & -5 & $g_{5}^{2}$ & & -1 & & \\
\hline$h_{1}^{1}$ & -2 & -2 & & 1 & $g_{5}^{3}$ & & 1 & & 1 \\
\hline$g_{2}^{0}$ & & -1 & & -1 & $h_{5}^{3}$ & & -1 & & -1 \\
\hline$g_{2}^{1}$ & & -3 & & -3 & $g_{5}^{4}$ & & -1 & & -1 \\
\hline$h_{2}^{1}$ & & & & -1 & $h_{5}^{4}$ & & -2 & & -2 \\
\hline$g_{2}^{2}$ & & -17 & & -15 & $g_{6}^{0}$ & & 3 & & 3 \\
\hline$h_{2}^{2}$ & & -16 & & -14 & $g_{6}^{1}$ & & -2 & & -2 \\
\hline$g_{3}^{0}$ & -3 & -31 & & -31 & $h_{6}^{1}$ & & 1 & & 1 \\
\hline$g_{3}^{1}$ & & -3 & & -3 & $g_{6}^{2}$ & & -3 & & -3 \\
\hline$h_{3}^{1}$ & -1 & 16 & & 15 & $h_{6}^{2}$ & & -2 & & -1 \\
\hline$g_{3}^{2}$ & & 3 & & 3 & $g_{6}^{3}$ & & -2 & & -2 \\
\hline$h_{3}^{2}$ & & -1 & & -1 & $h_{6}^{3}$ & & 2 & & 2 \\
\hline$g_{3}^{3}$ & & & & 2 & $g_{6}^{4}$ & & 1 & & 1 \\
\hline$h_{3}^{3}$ & & 3 & & 3 & $h_{6}^{4}$ & & -1 & & -1 \\
\hline$g_{4}^{0}$ & & -3 & & -3 & $g_{6}^{5}$ & & -1 & & -1 \\
\hline$g_{4}^{1}$ & & 5 & & 5 & $h_{6}^{5}$ & & -1 & & \\
\hline$h_{4}^{1}$ & & -3 & & -3 & $h_{6}^{6}$ & & & & -1 \\
\hline$g_{4}^{2}$ & & 8 & & 8 & $g_{7}^{0}$ & -1 & -2 & & -3 \\
\hline$h_{4}^{2}$ & & 5 & & 5 & $g_{7}^{1}$ & & 1 & & 1 \\
\hline $\begin{array}{l}n_{4} \\
g_{4}^{3}\end{array}$ & & 2 & & 1 & $h_{7}^{1}$ & & 3 & & 3 \\
\hline$h_{4}^{3}$ & & -3 & & -3 & $g_{7}^{2}$ & & & & 1 \\
\hline$g_{4}^{4}$ & & 1 & & 1 & $h_{7}^{4}$ & & 1 & & 1 \\
\hline$h_{4}^{4}$ & & 2 & & 1 & $g_{8}^{0}$ & & -1 & & -1 \\
\hline$g_{5}^{0}$ & -1 & 9 & & 10 & $g_{8}^{1}$ & & 1 & & 1 \\
\hline$g_{5}^{1}$ & & -1 & & -1 & $g_{8}^{2}$ & & 1 & & 1 \\
\hline & & & & & $h_{8}^{3}$ & & -1 & & -1 \\
\hline
\end{tabular}

1) When the first order external coefficients were admitted in the analysis all the coefficients including the three external ones were exactly recovered within the limits of precision of the computer.

2) All values are rounded off to the nearest $\mathrm{nT}$. Blanks or missing coefficients indicate the value was $0.5 \mathrm{nT}$ or less.

type of analysis and the weighting, as seen in Table 1. The $X Y Z$ solution confines significant changes to only a very few coefficients. In fact, with the $\sin \theta$ weighting a peculiar result is obtained; none of the coefficients change significantly as shown by all the blanks under this column. The two $F$ solutions with different weights on the other hand, track each other quite well and many coefficients are changed. It is of interest to note that $\left(g_{3}^{0}\right)_{i}$ changes most for the $F$ solutions and that change is almost twice the largest change for the $X Y Z$ solutions, $\left(g_{1}^{0}\right)_{i}$. 
Table 2. Examples of the determination of the field from external sources.

\begin{tabular}{|c|c|c|c|c|c|}
\hline \multirow{2}{*}{ Author } & \multirow{2}{*}{ Epoch } & \multicolumn{3}{|c|}{$\begin{array}{l}\text { External coefficients } \\
\text { (nanotesla) }\end{array}$} & \multirow{2}{*}{ Comments } \\
\hline & & $g_{1}^{0}$ & $g_{1}^{1}$ & $h_{1}^{1}$ & \\
\hline SchmidT & 1885 & -186 & 241 & -4 & \multirow{2}{*}{$\begin{array}{l}\text { See discussion of these in CHAPMAN and } \\
\text { BARTELS (1940). }\end{array}$} \\
\hline BAUER & 1922 & -523 & 69 & 113 & \\
\hline $\begin{array}{l}\text { FANSELAU } \\
\text { et al. }(1964)\end{array}$ & 1945 & $\begin{array}{l}45.0 \\
44.5 \\
31.1\end{array}$ & $\begin{array}{r}-11.4 \\
-9.6 \\
-3.6\end{array}$ & $\begin{array}{l}29.0 \\
27.2 \\
27.2\end{array}$ & $\begin{array}{l}\text { For analysis carried out to order } 15- \\
\text { For analysis carried out to order } 10- \\
\text { For analysis carried out to order } 6- \\
\text { Based on the world magnetic charts derived } \\
\text { by VESTINE } \text { et al. }(1947 \text { a) } \\
\text { (In each case all external coefficients up to the } \\
\text { limit of the analyses were determined.) }\end{array}$ \\
\hline $\begin{array}{l}\text { VESTINE } \\
\quad \text { et al. }(1947 \mathrm{~b})\end{array}$ & 1945 & \multicolumn{3}{|c|}{$\begin{array}{l}\text { No specific values } \\
\text { were given in report } \\
\text { although they were } \\
\text { computed and could } \\
\text { easily be reconstructed. }\end{array}$} & $\begin{array}{l}\text { "In fact, though most values of } C_{n}^{m}, S_{n}^{m} \text { up to } \\
m=3 \text { and } n=3 \text { indicated a few percent of } \\
\text { the field to be of external origin, the value } \\
\text { of } C_{1}^{0} \text { was } 0.000 \text {. It should be the largest } \\
\text { fraction, yet our analysis for this compo- } \\
\text { nent gives an external part less than } 1 \% \text {. } \\
\text { In fact, our analysis probably does not } \\
\text { reveal the existence of any permanent } \\
\text { external source of field.", }\end{array}$ \\
\hline $\begin{array}{l}\text { HuRwitz } \\
\text { et al. (1966) }\end{array}$ & 1965 & -82 & -24 & 17 & $\begin{array}{l}\text { Computed all external coefficients out to } \\
\text { order } 12 \text {. }\end{array}$ \\
\hline CAIN (1966) & 1965 & 19 & -9 & -16 & $\begin{array}{l}\text { GSFC }(7 / 65) \text {. Cain expressed the result in } \\
\text { terms of direct field values rather than } \\
\text { coefficients so that the signs are reversed } \\
\text { here from that shown in his paper. }\end{array}$ \\
\hline
\end{tabular}

Note: Apparently the Cain analysis is the only one for which flattening of the earth was taken into account.

The most interesting result is the inability of the model without external coefficients to accommodate effectively the ring current effects. This is shown by a comparison of component residuals. Since the coefficients are perfectly recovered within the limits of precision of the computer when the three external coefficients are admitted in the solution, the rms residual for that case is effectively zero, whereas when the three external coefficients are not admitted and an $X Y Z$ solution is made with a weight of 1 the residuals are as follows: $X r m s=$ $84 \mathrm{nT}, Y r m s=12 \mathrm{nT}, Z r m s=46 \mathrm{nT}$, total $r m s=56 \mathrm{nT}$. The $r m s$ residual was not obtained for the $F$ solution, but there is no reason to think it would be better than the component results.

We should try to include, in the analysis, proper functional forms to account for all external sources that are large enough to be significant in the final result. As the data samples improve, a number of significant external sources should become evident. 
In the past, several attempts have been made to determine some external coefficients. Early analyses ascribed about three percent of the total potential to external potential, $V^{e}$, causes and about as much to a nonpotential part, $N$ (due to earth-air currents). These early results are in question, however, because of inaccuracies in the original data.

Without making an exhaustive effort at completeness a few examples of the determination of external coefficients are given in Table 2.

Great differences can be seen in the results obtained so far. It is evident that more work is needed in this area before definitive external coefficients can be included in descriptions of the geomagnetic field.

Mr. L. Hurwitz, now retired, made some of the calculations shown in Table 1.

\section{REFERENCES}

Alldredge, L.R., G.D. Van Voorhis, and T.M. Davis, A magnetic profile around the world, J. Geophys. Res., 68, 3679-3692, 1963.

Bullard, E.C., The removal of trend from magnetic surveys, Earth and Planet. Sci. Letters, 2 , 293-300, 1967.

CAIN, J.C., Models of the earth's magnetic field, in Radiation Trapped in the Earth's Magnetic Field, edited by B. McCormac, pp. 7-25, Gordon and Breach, New York, 1966.

Chapman, S. and J. Bartels, Geomagnetism, pp. 543-1049, Clarendon Press, Oxford, 1940.

Davis, W.M. and J.C. CAIN, Removal of DS from POGO satellite data, (Abs.), EOS, Am. Geophys. Union Trans., 54, 242, 1973.

Fabiano, E.B. and N.W. Peddie, Evaluation of POGO (8/69): Mathematical model of the earth's inner magnetosphere, J. Geophys. Res., 76, 3816-3819, 1971.

Fanselau, G., H. Kautzlegen, O. Lucke, P. Mauersberger, and K. Sellien, Die Darstelling des geomagnetischen Potentials zur Epoche 1945.0 durch eine Entwicklung nach Kugelfunctionen bis zur 15 Ordnung, Pure and App. Geophys., 57, 5-30, 1964.

Gauss, C.F., Allgemeine Theorie des Erdmagnetismus, in Resultate aus den Beobachtungen des Magnetischen Verin im jahre 1838 (reprinted in Werke, Band 5, 121-193, Koniglichen Gessellschaft der Wissenschaften, Gottingen 1877), 1839.

Hurwitz, L., A discrepancy of vertical intensity in models of the geomagnetic field based on scalar intensity, NOAA Tech. Rpt., ERL 255 ESL-27, 50, 1972.

Hurwitz, L., D.G. Knapp, J.H. Nelson, and D.E. Watson, Mathematical model of the geomagnetic field for 1965, J. Geophys. Res., 71, 2373-2383, 1966.

Kolesova, V.I. and E.P. Kropachev, Spherical analysis of the geomagnetic field of the 1965 epoch to $n=23$ from ground based data, I. Method, Geomag. and Aeron., 12, 780-784, 1972.

Kolesova, V.I. and E.P. Kropachev, Spherical harmonic analysis of the geomagnetic field for the 1965 epoch up to $n=23$ according to ground-based data, II. Results, Geomag. and Aeron., 13, 127-131, 1973.

MCDonald, K.L. and R.H. Gunst, An analysis of the earth's magnetic field from 1835 to 1965 , ESSA Tech. Rpt., IER 46 IES-1, 87, 1967.

REGAN, R.D., Worldwide magnetic anomalies from POGO and COSMOS Data, (Abs.), IAGA Bull., No. 34, 267, 1973.

Vestine, E.H., I. Lange, L. LaPorte, and W.E. Scott, The geomagnetic field, its description and analysis, Dept. of Terres. Mag. Rpt., No. 580, 390, 1947 a.

Vestine, E.H., L. LaPorte, I. Lange, C. Cooper, and W.C. Hendrix, Description of the earth's main magnetic field, 1905-1945, Dept. of Terres. Mag. Rpt., No. 578, 532, $1947 \mathrm{~b}$.

ZMudA, A.J., (ed.), World Magnetic Survey, IAGA Bull., No. 28, 206, 1971. 